\title{
Antibody response to DNA vaccine against H5N1 avian influenza virus in broilers immunized according to three schedules
}

\author{
Anna Stachyra1', Anna Góra-Sochacka1', Włodzimierz Zagórski-Ostoja', Ewelina Król² and \\ Agnieszka Sirko ${ }^{\boxplus}$ \\ IInstitute of Biochemistry and Biophysics, Polish Academy of Sciences, Warsaw, Poland; ${ }^{2}$ Department of Recombinant Vaccines, Intercollegiate \\ Faculty of Biotechnology, University of Gdansk and Medical University of Gdansk, Gdańsk, Poland
}

\begin{abstract}
Broiler type chickens were immunized intramuscularly with a DNA vaccine encoding hemagglutinin (HA) from H5N1 avian influenza virus. The chickens were divided into four groups: control group which was not immunized, a group which obtained only one dose, and two groups which were immunized twice, one group with a boost two weeks after the priming and the other four weeks. Blood samples were collected at several time points and the dynamics of the humoral response to the vaccine was studied. High level of anti-HA antibodies was detected only in the last two groups, that is in chickens immunized according to the prime-boost strategy, regardless of the schedule. An additional interesting observation of this study was detection of the cross-reactivity of an anti-H5 HA positive serum with $\mathrm{H} 5 \mathrm{~N} 2$ and H1N1 viruses, suggesting that the DNA vaccine tested can induce antibodies of a broad specificity.
\end{abstract}

Key words: Avian influenza, H5N1, DNA vaccine, broilers

Received: 30 May, 2014; revised: 19 June, 2014; accepted: 18 August, 2014; available on-line: 01 September, 2014

\section{INTRODUCTION}

Chickens are the main poultry species raised for meat, eggs and hobby. During the hundreds of years of breeding they have been subjected to intensive selection which could affect their immunological characteristics and susceptibility to avian influenza, a poultry disease of great importance (Alexander, 2007; Graham-Rowe, 2011). Although the threat of an H5N1 influenza outbreak is now less imminent than during the 2006 epidemic, it is still real. Construction of an effective vaccine against avian influenza is a challenging task due to the continuous threat of new outbreaks caused by new subtypes, like H7N9, which occurred recently in China (Gao et al., 2013b). Therefore, not only the control and surveillance of the influenza virus spread is important (Capua \& Cattoli, 2013) but there is also an unquestionable need for modern veterinary vaccines that could be prepared without handling live viruses and would be consistent with DIVA European strategy (differentiating infection in vaccinated animals). DNA vaccines can potentially fulfill these criteria. In most experimental DNA vaccines for chickens two vaccine doses are administered one week (Mahmood et al., 2007; Song et al., 2010) or two weeks (Li et al., 2003; Li et al., 2013) apart, with the first dose applied at various age. The studies were often performed with SPF (specific pathogen-free) chickens, most frequently Leghorn White, which is a layer type breed (Yang et al., 2009; Chen et al., 2011; Gao et al., 2013a). Modern broiler lines, like Ross 508 (Mahmood et al., 2007; Song et al., 2013) or Chine's Yellow Hair chickens, a traditional Asian meat breed (Xu et al., 2008; Song et al., 2010) have been used much less frequently in such experiments. Other breeds, like Rhode Island Red, were tested only sporadically (Haygreen et al., 2006). All of these breeds have different metabolism, life span or body weight. Differences in optimal vaccination protocols for these lines could be thus anticipated.

The present study is part of a broader research project focused on chicken immunity and vaccination against avian influenza. The main aim of this study was on analysis of antigen-specific humoral response to a DNA vaccine encoding hemagglutinin from the $\mathrm{H} 5 \mathrm{~N} 1$ influenza virus isolated in Poland from a wild swan, using the broiler line Ross 308 most popular in the poultry industry in Poland. The immunological response of these broilers to the DNA vaccine administered according to three different schedules was studied in order to establish an optimal vaccination program.

\section{MATERIALS AND METHODS}

DNA vaccine. Construction of the plasmid for DNA vaccination with inserted synthetic DNA encoding HA from the H5N1 A/swan/Poland/305-135V08/2006 (EpiFluDatabase [http://platform.gisaid.org]; Accession No. EPI156789), vaccine preparation and chicken immunization were described previously (Stachyra et al., 2014). Briefly, the HA gene was optimized to the domestic chicken codon bias and engineered to contain deletion of the proteolytic cleavage site (from Arg-341 to Arg-346). Next, it was cloned into the pCI mammalian expression vector (Promega $\mathrm{GmbH}$, Germany) under control of CMV promoter. A dose of $125 \mu \mathrm{g}$ of plasmid DNA mixed with $20 \mu \mathrm{l}$ of Lipofectin transfection reagent (Life Technologies, USA) was used for each immunization.

Immunizations. Broiler (Ross 308) chickens were housed in cages in an experimental poultry house under standard commercial conditions. The vaccine was administered intramuscularly. The schedule of immuniza-

e-mail: asirko@ibb.waw.pl

Abbreviations: ELISA, enzyme-linked immunosorbent assay; HA, hemagglutinin; IPMA, immunoperoxidase monolayer assay; MDCK, Madin-Darby canine kidney cells. 
A

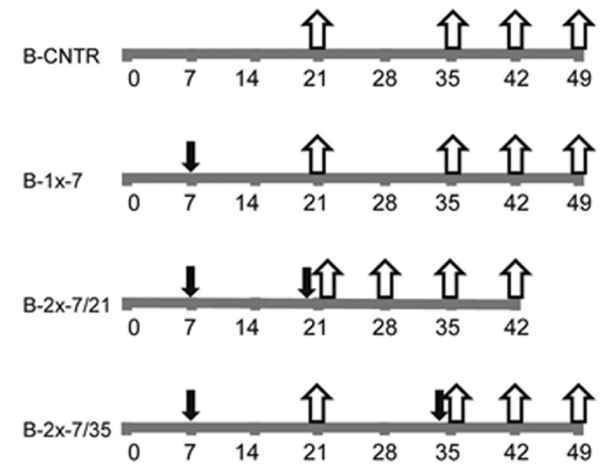

$\mathrm{B}$

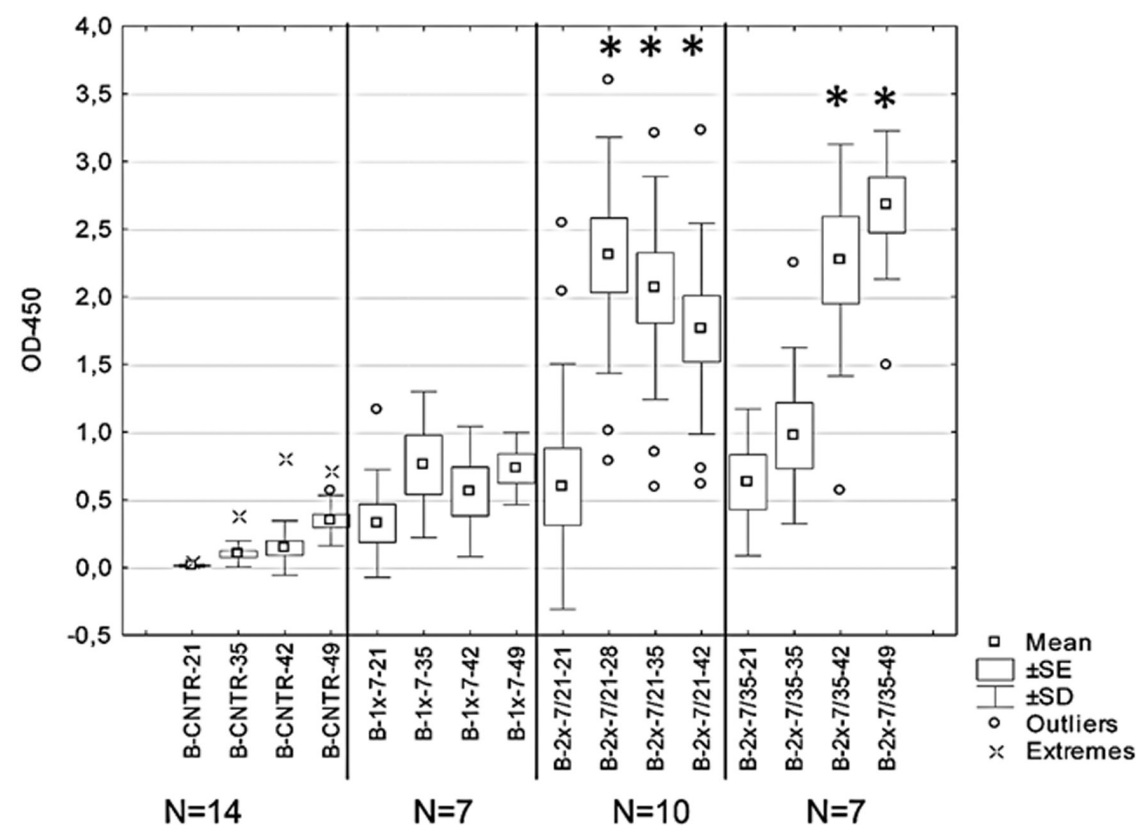

Figure 1. Dynamics of anti-H5 HA IgY level in sera of immunized chickens.

(A) Schedule of immunization. The immunization (black arrows) and blood collection (white arrows) are indicated above the respective day of life of the chickens from each group. (B) ELISA results for sera collected on the indicated days after hatching (21, $28,35,42$ and 49) calculated for group with standard errors $( \pm S E)$ and standard deviations $( \pm S . D$.) indicated. Asterisks denote mean values statistically different from the corresponding mean in the control group (CNTR). Chickens were immunized on day 7 (B-1x-7), 7 and 21 (B-2x-7/21), or 7 and 35 (B-2x-7/35). Number (N) of chickens per group is indicated.

tions and blood collections is shown in Fig. 1A. Shortly, broilers were divided into four groups ( $\mathrm{n}=7-14)$ : (1) BCNTR - control which did not receive any treatment, (2) B-1x-7 — immunized once on day 7 , (3) B-2x-7/21 - immunized twice on days 7 and 21 , (4) B-2x-7/35 immunized twice on day 7 and 35 . The experiment have been approved by the II Local Ethical Committee for Animal Experiments at Medical University of Warsaw, permit number 17/2009. All efforts were made to minimize suffering. At the end of the experiment the birds were humanely euthanized by decapitation.

Ezyme-linked immunosorbent assay (ELISA). Indirect ELISA for detection of anti-HA antibodies in serum was performed in 96-well polystyrene plates (MediSorp, Nunc, Denmark) as described previously (Stachyra et al., 2014). Plates were coated overnight with $50 \mu \mathrm{l}$ of affinity-purified, recombinant H5 HA (17-530, $\Delta 341-346$,
6xHis-tag at C-terminus) antigen (A/swan/Poland/305135V08/2006 (H5N1)) produced in a baculovirus system by Oxford Expression Technologies, UK, diluted in PBS to $6 \mu \mathrm{g} / \mathrm{ml}$. Goat anti-chicken $\operatorname{IgY}$ (Fc specific)-HRP IgGs (Pierce/Thermo Scientific, IL, USA) were used as secondary antibodies. All sera were diluted 200-folds.

Cells and influenza viruses. Madin-Darby canine kidney cells (MDCK) (Collection of the Department of Recombinant Vaccines, University of Gdansk, Poland) were cultured in Dulbecco's Modified Eagle's Medium (D-MEM) (Sigma-Aldrich, USA), supplemented with $10 \%$ heat-inactivated fetal bovine serum (FBS), 2 mM Lglutamine, $0.2 \%$ bovine serum albumin, $25 \mathrm{mM}$ HEPES buffer, $100 \mathrm{U} / \mathrm{ml}$ penicillin, $100 \mu \mathrm{g} / \mathrm{ml}$ streptomycin, at $37^{\circ} \mathrm{C}$ under $5 \% \mathrm{CO}_{2}$.

The avian influenza virus A/ostrich/Denmark/725/96 (H5N2) (kindly provided by R. Manvell, AHVLA, Wey- 
A

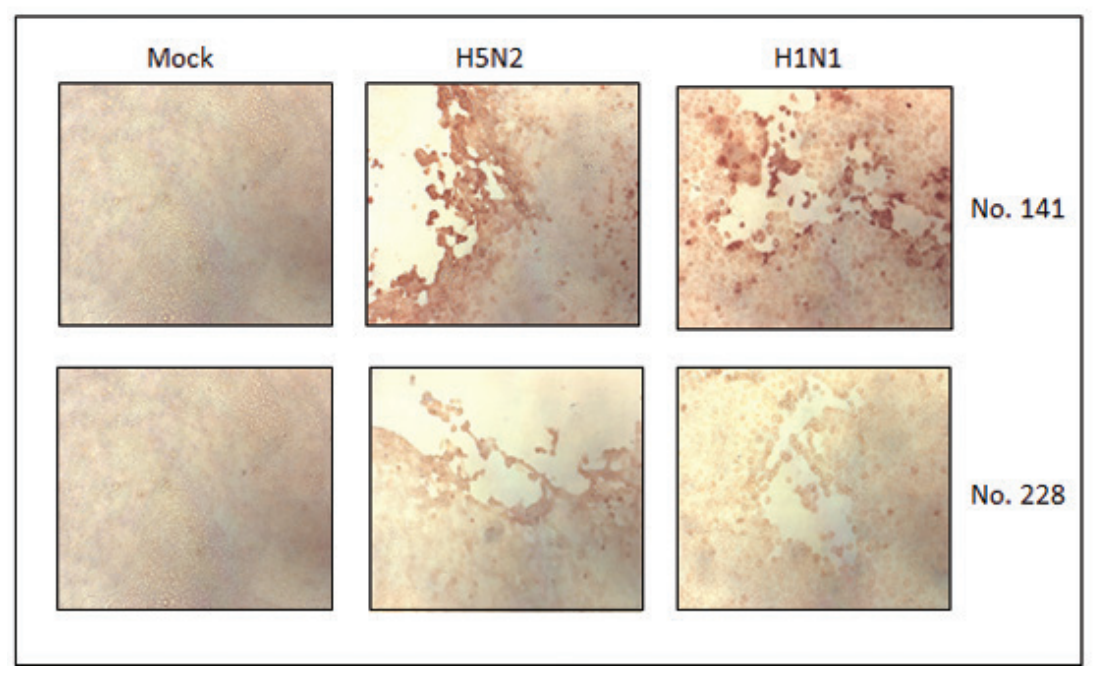

B

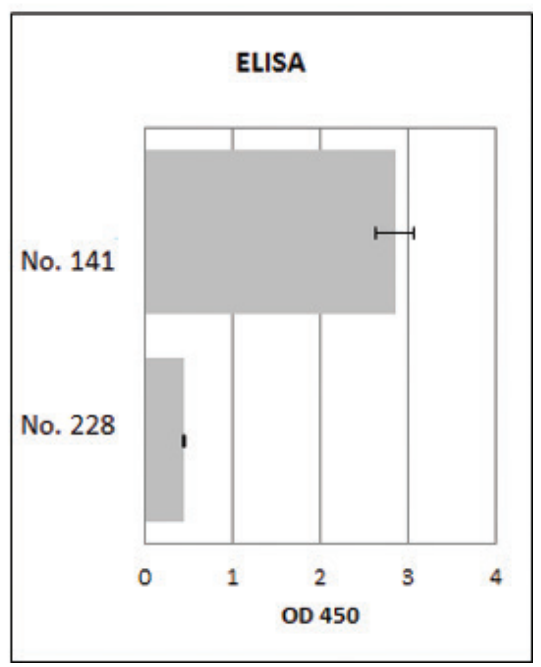

Figure 2. Cross-reactivity of the positive serum with a high anti-HA titer with two viral strains.

Chickens were immunized according to scheme B-2x-7/21 and sera collected on day 42 were used. For IPMA test, MDCK cells were mock infected or infected with avian influenza virus A/ostrich/Denmark/725/96 (H5N2) or isolate 32U of the pandemic human influenza A/ $\mathrm{H} 1 \mathrm{~N} 1$ virus. At $1 \mathrm{~h}$ post infection, unbound virus was removed and cells were incubated with overlay medium. Three days post infection cells were fixed and immunostained using the indicated sera (No. 141 - sample positive in ELISA test or No. 228 - negative control) to detect influenza A virus-infected cells. The results of IPMA test (A) and the results of ELISA test of the same samples (B) are shown.

bridge, UK) and human influenza virus (isolate 32U of the pandemic human influenza A/H1N1 virus) from the collection of the Department of Recombinant Vaccines, University of Gdansk, Poland were propagated in MDCK cells in the presence of $2 \mu \mathrm{g} / \mathrm{ml}$ TPCK (L-1-tosylamide-2-phenylethyl chloromethyl ketone) - trypsin (Sigma-Aldrich, USA). Viral stocks were stored at $-70^{\circ} \mathrm{C}$ and titrated by plaque assay before use.

Immunoperoxidase monolayer assay (IPMA). MDCK cell monolayers in 12-well plates were infected for $1 \mathrm{~h}$ at $37^{\circ} \mathrm{C}$ with either avian influenza virus $\mathrm{A} / \mathrm{os}-$ trich/Denmark/725/96 (H5N2) or isolate 32U of the pandemic human influenza $\mathrm{A} / \mathrm{H} 1 \mathrm{~N} 1$ virus. Unbound viral particles were removed by washing with serum-free medium, and the cell monolayers were overlaid with fresh serum-free medium containing 1.2\% Avicel (FMC BioPolymer, USA) and $2 \mu \mathrm{g} / \mathrm{ml}$ TPCK-trypsin. After 3 days, cells were washed with phosphate-buffered saline (PBS), fixed with 4\% paraformaldehyde and virusinfected cells were immunostained by incubating for 1 $\mathrm{h}$ with collected sera diluted 1:8 in PBS containing 1\% Tween 20 and 5\% fetal bovine serum (FBS). Anti-chicken horseradish peroxidase (HRP)-conjugated antibody (Santa Cruz Biotechnology, USA) was used as secondary antibody (diluted 1:1000 in PBS containing 1\% Tween 20 and $5 \% \mathrm{FBS})$. Infected cells were detected using the Vector Nova-Red kit (Vector Laboratories Ltd., UK). The infected cells appeared as red plaques on unstained background of uninfected cells.

Statistical analysis. For comparison of ELISA results in different groups the ANOVA Tukey HSD (Honestly Significant Difference) test for unequal $\mathrm{n}$ was applied, using STATISTICA 6.0 (Statsoft, Poland). The results were considered significant for $\mathrm{p}<0.05$.

\section{RESULTS AND DISCUSSION}

Three groups of broiler chickens were immunized intramuscularly either once or twice with $125 \mu \mathrm{g}$ of DNA vaccine per dose and the level of anti-HA antibodies in sera of the immunized chickens was determined by the ELISA test at different time points. The experiments were performed in poultry house cages, using standard commercial conditions, which allowed us to observe natural reactions to the immunization. The study lasted 6 weeks, which corresponds to the broiler life span in commodity production. The broiler chickens grow very fast and have intensive metabolism. Therefore, the immunization was started as early as possible; the first dose was administered on the seventh day after hatching. The second dose (if applied) was administered, depending on the group, on day 21 or 35 . The ELISA test demonstrated clear differences between the groups. The mean values of the levels of anti-HA antibodies in the experimental groups are shown in Fig. 1B. They were higher in the group immunized once than in the control group, but the difference was statistically nonsignificant. The second vaccination (booster) resulted in an increased humoral response regardless of the booster time. A statistically significant increase of the anti-HA antibody titer was observed one week after the second dose in both groups that were immunized twice. The anti-HA level remained high in those groups until the end of the experiment (42 or 49 days). These results indicate that two doses of the experimental DNA vaccine were required to elicit a high level of anti-HA antibodies. Interestingly, large biological variation was observed among responders within the experimental groups (Fig. 1B). Similar substantial inter-individual variation has been described in other DNA vaccine studies with chickens (Shan et al., 2011), which could be related to differences in DNA uptake by muscle fibers.

A considerable number of publications report chicken immunization with DNA vaccines, especially with avian influenza antigens. Such studies sometimes included measurement of IgY humoral response in serum samples and analysis of its dynamics (Jalilian et al., 2010; Liniger et al., 2012), but most of them concentrated on hemag- 
glutinin inhibition tests and virus neutralization assays (Jiang et al., 2007; Shan et al., 2011; Lim et al., 2012). In this study, the specificity of the anti-HA antibodies induced by our experimental DNA vaccine was evaluated preliminarily using the IPMA test which enables detection of cells exposing HA on their surface, such as cells infected by the virus. Two serum samples collected at day 42, one from a non-immunized chicken (negative in ELISA, No. 228) and one from a positive individual with a high titer of antibodies, immunized twice on days 7 and 21 (No. 141) were analyzed. The results clearly indicated that the serum negative in ELISA remained negative in the IPMA test, while the positive serum with a high anti-HA titer was able to detect cells infected by the H5N2 (A/ostrich/Denmark/725/96) virus (Fig. 2). Interestingly, the positive serum also detected cells infected by a serologically distant pandemic H1N1 strain of human influenza A virus, which apparently indicates the presence of broad-specificity antibodies in the serum (Fig. 2). The discovered cross-reactivity and presumed ability of our experimental DNA vaccine to induce antibodies with broad specificity is a very interesting observation justifying further detailed studies.

\section{Acknowledgements}

We thank Anna Doraczyńska-Filipczak for excellent veterinary support and Katarzyna Florys (IBA), Józef Kapusta (IBA), Katarzyna Szatraj (IBB) for their help and cooperation during immunization experiments and immunological analysis.

\section{Acknowledgments of financial support}

This work was supported by Innovative Economy Program, Grant No. WND-POIG.01.01.02-00-007/08.

\section{REFERENCES}

Alexander DJ (2007) An overview of the epidemiology of avian influenza. Vacine 25: 5637-5644.

Capua I, Cattoli G (2013) Prevention and control of highly pathogenic avian influenza with particular reference to H5N1. Virus Res 178: $114-120$.

Chen HY, Zhang HY, Li XS, Cui BA, Wang SJ, Geng JW, Li K (2011) Interleukin-18-mediated enhancement of the protective effect of an infectious laryngotracheitis virus glycoprotein B plasmid DNA vaccine in chickens. J Med Microbiol 60: 110-116.

Gao H, Li K, Gao L, Qi X, Gao Y, Qin L, Wang Y, Wang X (2013a) DNA prime-protein boost vaccination enhances protective immunity against infectious bursal disease virus in chickens. Vet Microbiol 164: 9-17.

Gao R, Cao B, Hu Y, Feng Z, Wang D, Hu W, Chen J, Jie Z, Qiu H, Xu K, Xu X, Lu H, Zhu W, Gao Z, Xiang N, Shen Y, He Z, Gu Y, Zhang Z, Yang Y, Zhao X, Zhou L, Li X, Zou S, Zhang
Y, Yang L, Guo J, Dong J, Li Q, Dong L, Zhu Y, Bai T, Wang S, Hao P, Yang W, Han J, Yu H, Li D, Gao GF, Wu G, Wang Y, Yuan Z, Shu Y (2013b) Human infection with a novel avian-origin influenza A (H7N9) virus. N Engl J Med 368:1888-1897.

Graham-Rowe D (2011) Epidemiology: Racing against the flu. Nature 480: S2-S3.

Haygreen EA, Kaiser P, Burgess SC, Davison TF (2006) In ovo DNA immunisation followed by a recombinant fowlpox boost is fully protective to challenge with virulent IBDV. Vaccine 24: 4951-4961.

Jalilian B, Omar AR, Bejo MH, Alitheen NB, Rasoli M, Matsumoto S (2010) Development of avian influenza virus H5 DNA vaccine and MDP-1 gene of Mycobacterium bovis as genetic adjuvant. Genet Vaccines Ther 8: 4.

Jiang Y, Yu K, Zhang H, Zhang P, Li C, Tian G, Li Y, Wang X, Ge J, Bu Z, Chen H (2007) Enhanced protective efficacy of H5 subtype avian influenza DNA vaccine with codon optimized HA gene in a pCAGGS plasmid vector. Antiviral Res 75: 234-241.

Li J, Huang Y, Liang X, Lu M, Li L, Yu L, Deng R (2003) Plasmid DNA encoding antigens of infectious bursal disease viruses induce protective immune responses in chickens: factors influencing efficacy. Virus Res 98: 63-74.

Li K, Gao H, Gao L, Qi X, Gao Y, Qin L, Wang Y, Wang X (2013) Adjuvant effects of interleukin-18 in DNA vaccination against infectious bursal disease virus in chickens. Vaccine 31: 1799-1805.

Lim KL, Jazayeri SD, Yeap SK, Alitheen NB, Bejo MH, Ideris A, Omar AR (2012) Co-administration of avian influenza virus H5 plasmid DNA with chicken IL-15 and IL-18 enhanced chickens immune responses. BMC Vet Res 8: 132.

Liniger M, Summerfield A, Ruggli N (2012) MDA5 can be exploited as efficacious genetic adjuvant for DNA vaccination against lethal H5N1 influenza virus infection in chickens. PLoS One 7: e49952.

Mahmood MS, Hussain I, Siddique M, Akhtar M, Ali S (2007) DNA vaccination with VP2 gene of very virulent infectious bursal disease virus (vvIBDV) delivered by transgenic E. coli DH5alpha given orally confers protective immune responses in chickens. Vaccine 25 : $7629-7635$

Shan S, Jiang Y, Bu Z, Ellis T, Zeng X, Edwards J, Tian G, Li Y, Ge J, Chen H, Fenwick S (2011) Strategies for improving the efficacy of a H6 subtype avian influenza DNA vaccine in chickens. J Virol Methods 173: 220-226.

Song H, Qiu B, Yan R, Xu L, Song X, Li X (2013) The protective efficacy of chimeric SO7/IL-2 DNA vaccine against coccidiosis in chickens. Res Vet Sci 94: 562-567.

Song H, Song X, Xu L, Yan R, Shah MA, Li X (2010) Changes of cytokines and IgG antibody in chickens vaccinated with DNA vaccines encoding Eimeria acervulina lactate dehydrogenase. Vet Parasitol 173: 219-227.

Stachyra A, Góra-Sochacka A, Sawicka R, Florys K, Sączyńska V, Olszewska M, Pikuła A, Śmietanka K, Minta Z, Szewczyk B, Zagórski W, Sirko A (2014) Highly immunogenic prime-boost DNA vaccination protects chickens against challenge with homologous and heterologous H5N1 virus. Trials Vaccinol 3: 40-46.

Xu Q, Song X, Xu L, Yan R, Shah MA, Li X (2008) Vaccination of chickens with a chimeric DNA vaccine encoding Eimeria tenella TA4 and chicken IL-2 induces protective immunity against coccidiosis. Vet Parasitol 156: 319-323.

Yang T, Wang HN, Wang X, Tang JN, Gao R, Li J, Guo ZC, Li YL (2009) Multivalent DNA vaccine enhanced protection efficacy against infectious bronchitis virus in chickens. J Vet Med Sci 71: 1585-1590. 